J. Dairy Sci. 92:617-620

doi:10.3168/jds.2008-1150

(c) American Dairy Science Association, 2009.

\title{
Short communication: Rising and lying behavior of heifers before and after alimentary oligofructose overload
}

\author{
D. B. Niss, ${ }^{\star}$ M. S. Herskin, ${ }^{* 1}$ A. M. Danscher, $\dagger$ and M. B. Thoefner \\ ${ }^{*}$ The University of Aarhus, Faculty of Agricultural Sciences, Institute of Animal Health, Welfare and Nutrition, Research Center Foulum, \\ PO Box 50, 8830 Tjele, Denmark \\ †University of Copenhagen, Faculty of Life Sciences, Institute of Large Animal Sciences, Medicine \& Surgery, Hoejbakkegaards Allé 5, \\ DK-2630 Taastrup, Denmark
}

\section{ABSTRACT}

The aim of this experiment was to describe the rising and lying behavior of heifers in the first $5 \mathrm{~d}$ after alimentary oligofructose overload to 1 ) acquire information about consequences of this experimental disease model in terms of animal behavior; and 2) contribute to the evaluation of the influence of disease on cattle welfare. Eight tethered Holstein-Friesian heifers were used and their behavior was observed for $10.5 \pm 2.7$ hours of light per day. Occurrence of rising and lying behavior and parts thereof were recorded. Clinical and physiological examinations were conducted throughout to confirm presence of ruminal acidosis and lameness. The duration of pre-lying intention movements were increased on d 3 after oligofructose overload compared with d -1 [28 (20 to 40 ) vs. 13 (10 to 16) s, respectively], and the duration of the total lying sequence was increased on $\mathrm{d} 1$ and 3 compared with the control day [27 (22 to 33 ) and 36 (25 to 48 ) vs. 17 (13 to 21 ) s, respectively]. The frequency and duration of getting up did not differ between days. These results suggest that disease induced by oligofructose overload (i.e., ruminal acidosis and lameness) can affect lying behavior in heifers as shown by increased duration of lying intentions and difficulty in lying down, which may indicate that lying motivation was thwarted, possibly by pain or discomfort.

Key words: rising behavior, oligofructose, lameness, ruminal acidosis

Hoof and leg problems in dairy cows are often long lasting. These problems are most likely associated with pain and are considered a significant welfare problem (Ingvartsen et al., 2006). Consequences of lameness in terms of behavioral welfare indicators have only recently been addressed and, until now, focus was on

Received March 5, 2008.

Accepted October 1, 2008.

${ }^{1}$ Corresponding author: MetteS.Herskin@agrsci.dk measures of activity and lying time (Juarez et al., 2003; O'Callaghan et al., 2003). From other animal species it is known that disease can lead to behavioral changes such as immobility and recumbency, which function to conserve energy and encourage recovery (Hart, 1988), but very few descriptions of sickness or pain behavior exist in cattle (Borderas et al., 2008). Based on studies in clinically healthy animals, lying behavior has a high priority in cattle (Jensen et al., 2005; Munksgaard et al., 2005). Because lying down is a prerequisite thereof, it is considered an important behavioral indicator during evaluation of diseases in terms of animal welfare.

Thoefner et al. (2004) described a model in which alimentary oligofructose overload was used to induce development of ruminal acidosis followed by clinical signs of lameness. This model allowed scientific study of sickness behavior in cattle and was used in the present experiment. The aim was to describe rising and lying down behavior of heifers in the initial $5 \mathrm{~d}$ after alimentary oligofructose overload to 1) acquire information about consequences of the experimental model in terms of animal behavior; and 2) contribute to the evaluation of the influence of disease on cattle welfare. The present behavioral study was conducted as part of a larger experiment undertaken to examine the pathophysiology of lameness in heifers given an oral bolus of oligofructose (Danscher et al., 2009).

Eight clinically healthy, nonpregnant, Danish Holstein-Friesian heifers with a BW of 276 to $443 \mathrm{~kg}$ (371 $\pm 55 \mathrm{~kg}$, mean $\pm \mathrm{SE})$ and aged 15 to $28 \mathrm{mo}(22.8 \pm 4.8$ mo, mean $\pm \mathrm{SE}$ ) from a commercial herd were used. Before the experiment, the heifers were managed in groups of 4 to 12 on deep bedding for at least 6 mo. For practical reasons the animals were allocated randomly to 2 blocks of 4 animals. The first block took place in June 2006 and the second in August 2006.

During the experiment the heifers of each block were kept in tie stalls in a separate room in an experimental barn. The barn was lit with natural light. The heifers were moved to the experimental barn at least $2 \mathrm{wk}$ before initiation of the experiment, and were kept in 
Table 1. Ethogram of behavior recorded before and after oligofructose overload

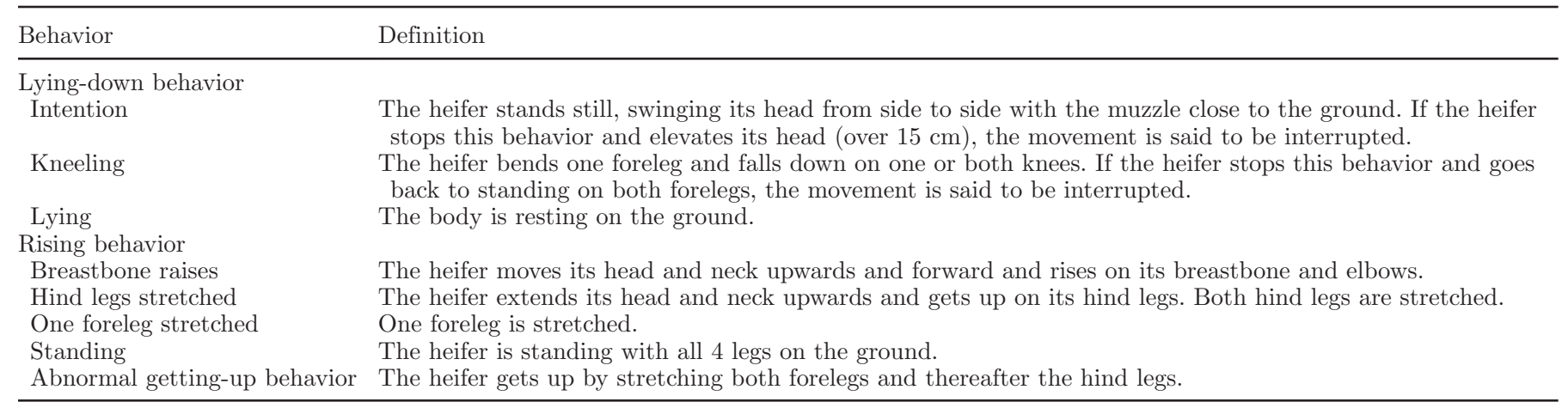

tie-stalls (by a chain of $88 \mathrm{~cm}$ tied at a height of 20 $\mathrm{cm}$ above ground). Each tie-stall measured $182 \times 143$ $\mathrm{cm}$ with a neck bow at a height of $93 \mathrm{~cm}$. The stalls were provided with 3 to $4 \mathrm{~cm}$ of sawdust. Each heifer had free access to water from a water bowl and was fed grass hay twice daily at 0800 and $1500 \mathrm{~h}$ for ad libitum intake.

The experiment consisted of daily observations of behavior from $1 \mathrm{~d}$ before $(\mathrm{d}-1)$ until $5 \mathrm{~d}$ after oligofructose overload. The experiment was planned and performed in a manner to prevent any unnecessary pain and discomfort for the animals. All animals were cared for and treated according to a protocol approved by the Danish Animal Experiments Inspectorate (file no. 2006/561-1132) before performance.

The heifers were given a carbohydrate overload consisting of oligofructose (Beneo P95, Orafti Active Food Ingredients, Tienen, Belgium). Powdered oligofructose was dissolved in warm water and administered via tube-feeding directly into the reticulum/rumen using an induction bolus dose of $17 \mathrm{~g} / \mathrm{kg}$ of BW. A priming dose of $5 \%$ of the main induction dose was given twice daily for $3 \mathrm{~d}$ before the actual overload to gradually allow adjustment of the forestomach microbiota to the new source of carbohydrate. In the morning of $d 0$ (at approximately $1000 \mathrm{~h}$ ) the fructan overloads were given to the heifers in a process taking approximately $10 \mathrm{~min}$ per individual. Supportive intravenous fluid therapy was given to the heifers to alleviate metabolic acidosis and dehydration. Calcium borogluconate (14 mg of Ca/ $\mathrm{mL})$ was given at $1.4 \mathrm{~mL} / \mathrm{kg}$ of BW. Bicarbonate $(84$ $\mathrm{g} / \mathrm{L}$ ) was given in a quantity of $1.5 \mathrm{~mL} / \mathrm{kg}$ of $\mathrm{BW}$, and Ringer's acetate (Fresenius Kabi, Uppsala, Sweden) was given in a quantity of $15 \mathrm{~mL} / \mathrm{kg}$ of BW. All heifers were given all 3 solutions at $1800 \mathrm{~h}$ on the day of the overload. Six hours later, all heifers received only bicarbonate and Ringer's acetate solutions.

Throughout the experiment, the heifers were subjected to daily walking and trotting by hand and were scored for gait and lameness using the scale of Sprecher et al. (1997) as well as an American Association of Equine Practitioners (www.aaep.org) scale.

The heifers were catheterized for blood sampling in the aural vein on $d-7$. From $d-3$ heifers were subject-

Table 2. Behavioral variables used to compared effects of oligofructose overload

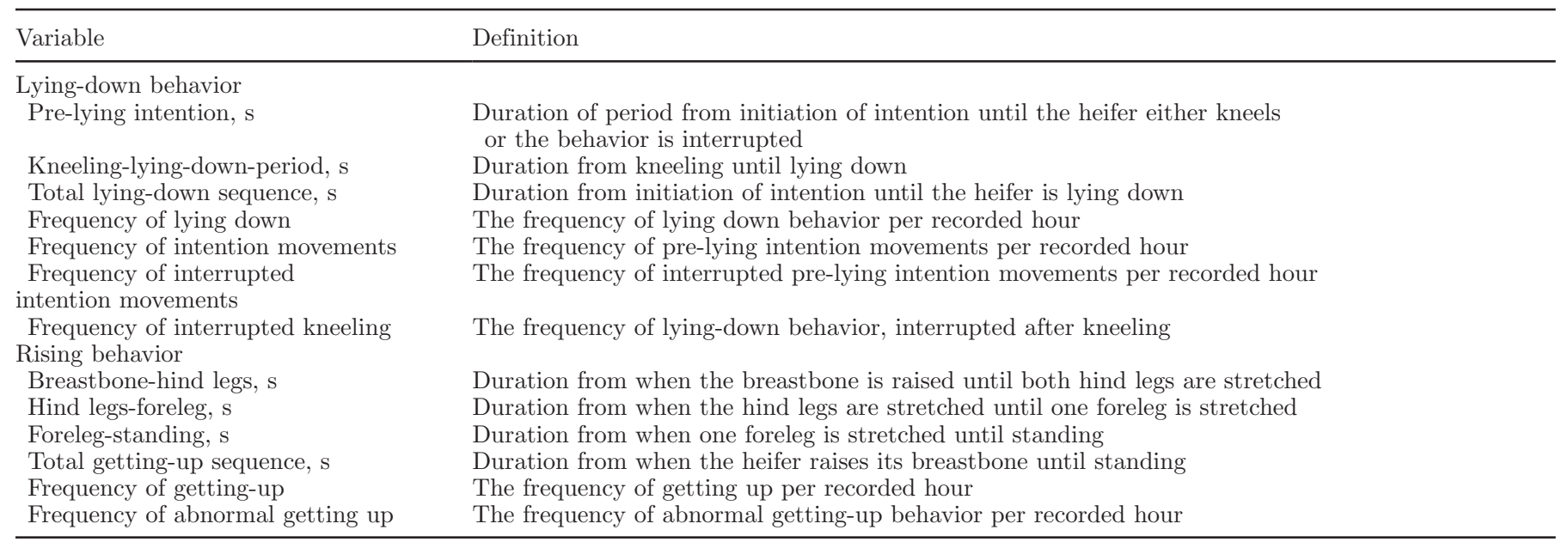


ed to clinical and physiological examinations according to a predefined protocol. Examinations were carried out every $24 \mathrm{~h}$ until the carbohydrate overload, after which examinations took place every $6 \mathrm{~h}$ for the next $48 \mathrm{~h}$. From d 2 to 5 , the heifers were examined every $24 \mathrm{~h}$. Two individuals handled the heifers at examination times. Further details of the main experiment are described by Danscher et al. (2009).

All occurrences of rising and lying-down behavior were recorded using time-lapse video recording. An ethogram of the recorded behavior is shown in Table 1. Recording was only possible during daylight (from approximately 0700 to $2000 \mathrm{~h}$ ), and all periods when research personnel were present within the stalls were excluded, leading to a mean recording period of 10.5 h/d (range: 4.8 to $12.8 \mathrm{~h} / \mathrm{d}$ ). Calculated behavioral variables are in Table 2 .

One heifer's reaction to the oligofructose overload was markedly more severe than that of the others. Despite intensive aggressive supportive therapy and close observation, she died during the experiment and data from this animal were omitted. The duration of rising behavior was analyzed based on data from 5 heifers because no spontaneous rising behaviors were recorded from 2 of the heifers on d 1 and 3, respectively. Data from all 7 heifers were used in the analysis of the frequency of rising. Interrupted kneeling and abnormal lying-down and rising behavior were not treated statistically because of very low representation in the data set.

Because of the variable registration periods all frequencies were calculated as occurrence per hour. The oligofructose bolus was given at $1000 \mathrm{~h}$ on $\mathrm{d} 0$ and each 24-h period (day) started at $1000 \mathrm{~h}$, so that the control day, by definition, was initiated $24 \mathrm{~h}$ before the overload. Differences between days were tested using one-way repeated measures ANOVA (Sigmastat, Systat Software, version 3.1, San Jose, CA). The results are presented as means \pm SE. In cases of nonnormality, Friedman repeated measures ANOVA on ranks (Sigmastat, Systat Software, version 3.1) was used and the results given as medians followed by 25 to $75 \%$ quartiles. For both analyses, the Tukey test was used for all pairwise comparisons of the means or mean ranks of the treatment groups.

Spearman rank correlations of SAS (Version 8e, SAS Institute Inc., Cary, NC) were used to test for correlations between selected clinical and physiological traits for ruminal acidosis and lameness and 1) the duration of pre-lying intention movements; and 2) the duration of lying-down behavior on $\mathrm{d} 1$ and 3 (days differing from control period). The selected variables were rumen $\mathrm{pH}$ and contractions, rectal temperature, temperature of coronary band, and lameness scores. 
No differences between the days were found for the frequencies of lying-down events (overall mean: $0.4 \pm$ $0.2 / \mathrm{h}$ ). The duration of the total lying-down sequence was affected by treatment leading to a longer duration on d 1 compared with $\mathrm{d}-1[27$ (22 to 23 ) vs. 16 (13 to 21 ) s, respectively, $P<0.001$; Table 3], and a longer duration of lying-down on $\mathrm{d} 3$ than on $\mathrm{d}-1$ [36 (25 to 48 ) vs. 16 (13 to 21 ) s, respectively, $P<0.001$; Table 3], but the duration of the last part of lying-down (from kneeling to recumbency) did not differ between the days (overall mean: $7 \pm 5 \mathrm{~s}$ ). No differences were found between the days for the frequency of pre-lying intention movements (overall mean: $0.7 \pm 0.4 / \mathrm{h}$ ) or the frequency of interrupted unsuccessful intention movements (overall mean: $0.2 \pm 0.2 / \mathrm{h}$ ). But the heifers showed longer duration of pre-lying intention movements on d 3 compared d -1 [28 (20 to 40) vs. 16 (10 to 16$) \mathrm{s}$, respectively, $P=0.005$; Table 3]. A tendency was found for the frequency of rising to be higher on $\mathrm{d}$ 2 than on $\mathrm{d} 1(0.4 \pm 0.2 / \mathrm{h}$ vs. $0.2 \pm 0.2 / \mathrm{h}$, respectively; $P=0.053$, Table 3 ), but no differences were found for the duration of the rising sequence (overall mean: $9 \pm$ $12 \mathrm{~s}$ ). However, the duration of the first phase of the behavior (from breastbone rises and until hind legs are stretched) tended to be longer on $\mathrm{d} 1,2$, and 3 than on $\mathrm{d}-1(P=0.068$; Table 3$)$. No significant correlations between clinical or physiological traits and behavior were found.

The results of the present experiment suggest that lying motivation of heifers was thwarted during disease, as indicated by the longer duration of pre-lying intention movements on $\mathrm{d} 3$ than on $\mathrm{d}-1$ and a longer duration of lying down on $\mathrm{d} 1$ and 3 compared with $\mathrm{d}-1$. It is known that rising and lying-down behavior of cattle can be affected by housing (Lidfors, 1989; Krohn and Munksgaard, 1993), but this is one of the first descriptions of the influence of disease on this behavior. Krohn and Munksgaard (1993) showed that the lying and rising behavior of healthy cows in tie-stalls was different from the behavior of cows in loose housing systems, as the tied cows used more time to lie down and had longer periods of pre-lying intention movements. The present results were obtained with a limited number of animals, but suggest that oligofructose overload can change the behavior comparably. Both metabolic and leg problems appear to lead to a thwarting of the lyingdown motivation in cattle and potentially prevent the animals from lying down. This study, however, did not investigate the existence of discomfort or pain.

\section{REFERENCES}

Borderas, T. F., A. M. B. de Passillé, and J. Rushen. 2008. Behavior of dairy calves following a low dose of bacterial endotoxin. J. Anim. Sci. 86:2920-2927.

Danscher, A. M., J. M. D. Enemark, E. Telezhenko, N. Capion, C. T. Ekstrøm, and M. B. Thoefner. 2009. Oligofructose overload induces lameness in cattle. J. Dairy Sci. 92:607-616

Hart, B. L. 1988. Biological basis of the behaviour of sick animals. Neurosci. Biobehav. Rev. 12:123-137.

Ingvartsen, K. L., P. T. Thomsen, T. W. Bennedsgaard, and M. D. Rasmussen. 2006. Production diseases in cattle. Pages 77-105 in Welfare of Dairy Cows and Calves. L. Munksgaard, and E. Søndergaard, ed. DJF, Foulum, Tjele, Denmark.

Jensen, M. B., L. J. Pedersen, and L. Munksgaard. 2005. The effect of reward duration on demand functions for rest in dairy heifers and lying requirements as measured by demand functions. Appl. Anim. Behav. Sci. 90:207-217.

Juarez, S. T., P. H. Robinson, E. J. DePeters, and E. O. Price. 2003. Impact of lameness on behaviour and productivity of lactating Holstein cows. Appl. Anim. Behav. Sci. 83:1-14.

Krohn, C. C., and L. Munksgaard. 1993. Behaviour of dairy cows kept in extensive (loose housing/pasture) or intensive (tie stall) environments. Appl. Anim. Behav. Sci. 37:1-16.

Lidfors, L. 1989. The use of getting up and lying down movements in the evaluation of cattle environments. Vet. Res. Commun. $13: 307-324$.

Munksgaard, L., M. B. Jensen, L. J. Pedersen, S. W. Hansen, and L. Matthews. 2005. Quantifying behavioural priorities - Effects of time constraints on behaviour of dairy cows, Bos taurus. Appl. Anim. Behav. Sci. 92:3-14.

O'Callaghan, K. A., P. J. Cripps, D. Y. Downham, and R. D. Murray. 2003. Subjective and objective assessment of pain and discomfort due to lameness in dairy cows. Anim. Welf. 12:605-610.

Sprecher, D. J., D. E. Hostetler, and J. B. Kaneene. 1997. A lameness scoring system that uses posture and gait to predict dairy cattle reproductive performance. Theriogenology 47:1179-1187.

Thoefner, M. B., C. C. Pollitt, A. W. van Eps, G. J. Milinovich, D. J. Trott, O. Wattle, and P. H. Andersen. 2004. Acute bovine laminitis: A new induction model using alimentary oligofructose overload. J. Dairy Sci. 87:2932-2940. 